\title{
Efficient Synthesis of $\beta$-Enaminones and $\beta$-Enaminoesters Catalyzed by Gold (I)/Silver (I) under Solvent-Free Conditions
}

\author{
Ming Zhang ${ }^{1}$, Ablimit Abdukader ${ }^{1,2}$, Yong Fu ${ }^{1}$ and Chengjian Zhu ${ }^{1,3, *}$
}

1 State Key Laboratory of Coordination Chemistry, School of Chemistry and Chemical Engineering, Nanjing University, Nanjing 210093, China; E-Mails: chemliu13@gmail.com (M.Z.); dg1024002@smail.nju.edu.cn (A.A.); fuyong2008@163.com (Y.F.)

2 School of Chemistry and Chemical Engineering, Xinjiang University, Urumqi 830046, China

3 State Key Laboratory of Organometallic Chemistry, Shanghai Institute of Organic Chemistry, Chinese Academy of Sciences, Shanghai 200032, China

* Author to whom correspondence should be addressed; E-Mail: cjzhu@nju.edu.cn; Tel./Fax: +86-25-8359-4886.

Received: 15 January 2012; in revised form: 16 February 2012 / Accepted: 17 February 2012 / Published: 6 March 2012

\begin{abstract}
An efficient method for the synthesis of $\beta$-enaminones and $\beta$-enaminoesters using a combination of $\left[\left(\mathrm{PPh}_{3}\right) \mathrm{AuCl}\right] / \mathrm{AgOTf}$ as catalyst has been developed. The reaction between 1,3-dicarbonyl compounds and primary amines was carried out under solvent-free conditions with low catalyst loading in good to excellent yields at room temperature.
\end{abstract}

Keywords: gold; solvent-free; amines; $\beta$-enaminones; $\beta$-enaminoesters

\section{Introduction}

$\beta$-Enaminones and $\beta$-enaminoesters are highly useful building blocks [1-3], which can be further transformed into valuable natural therapeutic and biologically active compounds such as anticonvulsivant [4,5], anti-inflammatory [6], and antitumor agents [7,8]. Moreover, they are useful intermediates for the preparation of aminoesters [9], $\alpha, \beta$-aminoacids [10,11], peptides [12], quinolines [13,14], azocompounds [15,16] and alkaloids [17-19]. Owning to their significances in organic synthesis, considerable efforts have been dedicated to prepare $\beta$-enaminones and $\beta$-enaminoesters. One of the most straightforward methods is condensation between 1,3-dicarbonyls and amines under reflux conditions [20]. Other improved methods for this amination reaction were successively 
developed [21-34]. However, in these procedures, the long reaction time, high reaction temperatures, and high catalyst loadings required could limit their further applications in organic synthesis.

Gold (I) and gold (III) salts have emerged as versatile catalysts to facilitate new carbon-carbon or carbon-heteroatom bond formation in a variety of reactions [35-38]. In 2003, Arcadi reported that gold (III) derivatives could catalyze the condensation reaction of 1,3-dicarbonyls and ammonia/amines [27], however, when the aromatic amine had been involved in the reaction only $60 \%$ yield was obtained. With increasing attention being paid to economically simple and environmentally safe methods, the recent trends in organic reaction are oriented to solvent-free conditions [39-41]. Herein, we report a practical method for the synthesis of $\beta$-enaminones and $\beta$-enaminoesters under solvent-free conditions by using $\left[\left(\mathrm{PPh}_{3}\right) \mathrm{AuCl}\right] / \mathrm{AgOTf}$ as catalyst with lower catalyst loading at room temperature (Scheme 1).

Scheme 1. Gold (I)/silver (I) catalyzed enamination of $\beta$-dicarbonyl compounds.<smiles>[R]C(=O)C([R])C([R])=O</smiles><smiles>[R]NC([R])=C([R])C([R])=O</smiles>

\section{Results and Discussion}

Initially, the reaction between acetylacetone and 4-methoxyaniline was carried out without catalyst under solvent-free conditions at room temperature for $2 \mathrm{~h}$; only $25 \%$ yield of the desired product could be obtained (Table 1, entry 1). The salt $\left(\mathrm{PPh}_{3}\right) \mathrm{AuCl}(1 \mathrm{~mol} \%)$ indicated moderate catalytic activity (33\% yield) (Table 1 , entry 2$)$, while the silver salt AgOTf ( $1 \mathrm{~mol} \%$ ) afforded a lower yield (28\% yield) (Table 1, entry 3$)$. Surprisingly, by combining $\left(\mathrm{PPh}_{3}\right) \mathrm{AuCl}(1 \mathrm{~mol} \%)$ with $\operatorname{AgOTf}(1 \mathrm{~mol} \%)$ as cocatalyst, the product could be obtained in higher yield (98\%) than when a single salt was used (Table 1, entry 4), so $\left(\mathrm{PPh}_{3}\right) \mathrm{AuCl} / \mathrm{AgOTf}$ was chosen as a promising catalyst for the reaction. The reaction was found to be sluggish when dichloromethane (DCM, $1 \mathrm{~mL}$ ) was chosen as solvent (Table 1, entry 5). Various amines were examined in this enamination reaction with acetylacetone, and the corresponding $\beta$-enaminones were obtained in excellent yields $(85 \%-98 \%)$. The results are listed in Table 2.

Table 1. Screening of the reaction conditions for the enamination ${ }^{\mathrm{a}}$.

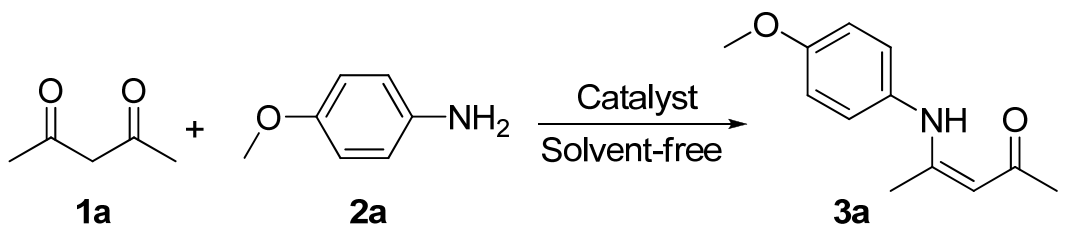

\begin{tabular}{llll}
\hline Entry & Catalyst & Time (h) & Yield (\%) \\
\hline 1 & - & 2 & 25 \\
2 & $\left(\mathrm{PPh}_{3}\right) \mathrm{AuCl}$ & 2 & 33 \\
3 & $\mathrm{AgOTf}$ & 2 & 28 \\
$\mathbf{4}$ & $\left(\mathrm{PPh}_{3}\right) \mathrm{AuCl}+\mathbf{A g O T f}$ & $\mathbf{2}$ & $\mathbf{9 8}$ \\
$5^{\mathrm{c}}$ & $\left(\mathrm{PPh}_{3}\right) \mathrm{AuCl}+\mathrm{AgOTf}$ & 6 & 85 \\
\hline
\end{tabular}

\footnotetext{
${ }^{\mathrm{a}}$ Reaction conditions: See typical procedure; ${ }^{\mathrm{b}}$ Isolated yield; ${ }^{\mathrm{c}}$ The reaction was carried out in DCM.
} 
Table 2. Synthesis of $\beta$-enaminones with $\left[\left(\mathrm{PPh}_{3}\right) \mathrm{AuCl}\right] / \mathrm{AgOTf}$ under solvent-free conditions ${ }^{\mathrm{a}}$.

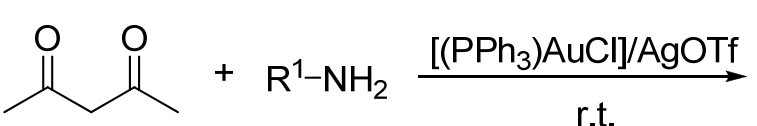

$1 \mathrm{a}$
2<smiles>[R1]N/C(C)=C/C(C)=O</smiles>

3

\begin{tabular}{|c|c|c|c|c|c|}
\hline Entry & $2\left(R^{1}\right)$ & Time & 3 & & Yield (\%) \\
\hline 1 & $4-\mathrm{CH}_{3} \mathrm{OC}_{6} \mathrm{H}_{4}$ & $2 \mathrm{~h}$ & & & 98 \\
\hline 2 & $\mathrm{C}_{6} \mathrm{H}_{5}$ & $4 \mathrm{~h}$ & & & 85 \\
\hline 3 & $4-\mathrm{CH}_{3} \mathrm{C}_{6} \mathrm{H}_{4}$ & $3 \mathrm{~h}$ & & & 87 \\
\hline 4 & $4-\mathrm{BrC}_{6} \mathrm{H}_{4}$ & $4 \mathrm{~h}$ & & & 90 \\
\hline 5 & $4-\mathrm{ClC}_{6} \mathrm{H}_{4}$ & $5 \mathrm{~h}$ & & & 88 \\
\hline 6 & $\mathrm{C}_{10} \mathrm{H}_{7}$ & $5 \mathrm{~h}$ & & & 96 \\
\hline 7 & $\mathrm{Bn}$ & $5 \mathrm{~min}$ & & $3 \sigma$ & 95 \\
\hline 8 & $\mathrm{n}-\mathrm{C}_{4} \mathrm{H}_{9}$ & $5 \mathrm{~min}$ & & $3 h$ & 96 \\
\hline 9 & Allyl & $1.5 \mathrm{~h}$ & & $3 \mathbf{i}$ & 98 \\
\hline 10 & 2-Hydroxyethyl & $5 \mathrm{~min}$ & & $3 \mathbf{j}$ & 96 \\
\hline
\end{tabular}

${ }^{\mathrm{a}}$ Reaction conditions: See typical procedure; ${ }^{\mathrm{b}}$ Isolated yield.

We then extended the scope of various amines with $\beta$-ketoesters using $1 \mathrm{~mol} \%$ loading of $\left[\left(\mathrm{PPh}_{3}\right) \mathrm{AuCl}\right] / \mathrm{AgOTf}$ catalyst, and the results are summarized in Table 3. All the desired products could be obtained in high yields (76-98\%). In addition, high chemoselectivity and regioselectivity can be obtained in this reaction since the ester group is less electrophilic than the keto carbonyl group; and only a single product was observed when the reaction was carried out between amine and $\beta$-ketoesters. Generally, the electronic properties of the aryl group appeared to slightly influence the reactivity. It is clear from our results that aromatic amines containing electron-donating groups like methoxyl and methyl (Table 3, entries 2 and 3) give the corresponding products in higher yields compared to the electron-withdrawing ones (Table 3, entries 4 and 5). Aliphatic amines (Table 3, entries 7-10) were more reactive than aromatic amines (Table 3, entries 1-6), and the reactions were completed within a 
shorter time. Furthermore, cyclic $\beta$-ketoesters could also afford the desired product in high yields (Table 3, entries 11-16).

Table 3. Synthesis of $\beta$-enaminoesters with $\left[\left(\mathrm{PPh}_{3}\right) \mathrm{AuCl}\right] / \mathrm{AgOTf}$ under solvent-free conditions ${ }^{\mathrm{a}}$.

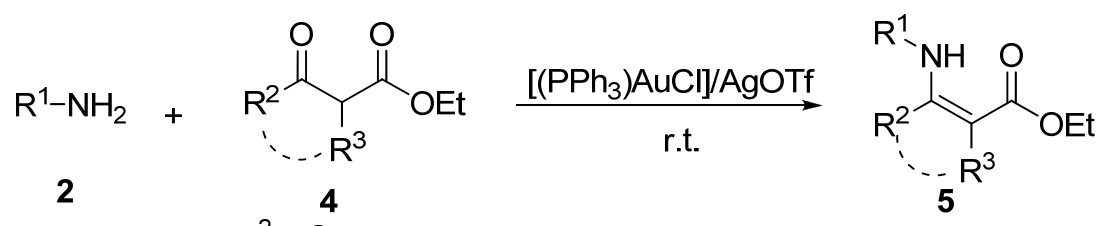

$$
\begin{aligned}
& \text { 4a } \mathrm{R}^{2}=\mathrm{CH}_{3}, \mathrm{R}_{3}=\mathrm{H} \\
& \text { 4b R } \mathbf{R}^{2}, \mathrm{R}^{3}=\left(\mathrm{CH}_{2}\right)_{3}
\end{aligned}
$$

\begin{tabular}{|c|c|c|c|c|c|c|}
\hline Entry & $2\left(R^{1}\right)$ & 4 & Time & 5 & & Yield (\%) ${ }^{b}$ \\
\hline 1 & $4-\mathrm{CH}_{3} \mathrm{OC}_{6} \mathrm{H}_{4}$ & $4 a$ & $3 \mathrm{~h}$ & & & 98 \\
\hline 2 & $\mathrm{C}_{6} \mathrm{H}_{5}$ & $4 a$ & $5 \mathrm{~h}$ & & & 82 \\
\hline 3 & 4- $\mathrm{CH}_{3} \mathrm{C}_{6} \mathrm{H}_{4}$ & $4 a$ & $4 \mathrm{~h}$ & & & 92 \\
\hline 4 & $4-\mathrm{BrC}_{6} \mathrm{H}_{4}$ & $4 a$ & $5 \mathrm{~h}$ & & & 86 \\
\hline 5 & $4-\mathrm{ClC}_{6} \mathrm{H}_{4}$ & $4 a$ & $5 \mathrm{~h}$ & & & 76 \\
\hline 6 & $\mathrm{C}_{10} \mathrm{H}_{7}$ & $4 a$ & $8 \mathrm{~h}$ & & & 85 \\
\hline 7 & $\mathrm{Bn}$ & $4 a$ & $5 \mathrm{~min}$ & & 50 & 97 \\
\hline 8 & $\mathrm{n}-\mathrm{C}_{4} \mathrm{H}_{9}$ & $4 a$ & $5 \mathrm{~min}$ & & $5 \mathrm{~h}$ & 95 \\
\hline 9 & Allyl & $4 a$ & $1 \mathrm{~h}$ & & $5 i$ & 97 \\
\hline 10 & 2-Hydroxyethyl & $4 a$ & $5 \mathrm{~min}$ & & & 96 \\
\hline 11 & $4-\mathrm{CH}_{3} \mathrm{OC}_{6} \mathrm{H}_{4}$ & $4 b$ & $2 \mathrm{~h}$ & & & 93 \\
\hline 12 & $\mathrm{C}_{6} \mathrm{H}_{5}$ & $4 b$ & $2 \mathrm{~h}$ & & & 87 \\
\hline 13 & 4- $\mathrm{CH}_{3} \mathrm{C}_{6} \mathrm{H}_{4}$ & $4 b$ & $1.5 \mathrm{~h}$ & & & 94 \\
\hline
\end{tabular}


Table 3. Cont.

\begin{tabular}{|c|c|c|c|c|c|}
\hline Entry & $2\left(R^{1}\right)$ & 4 & Time & 5 & Yield (\%) \\
\hline 14 & $4-\mathrm{BrC}_{6} \mathrm{H}_{4}$ & $4 b$ & $2 \mathrm{~h}$ & & 92 \\
\hline 15 & $4-\mathrm{ClC}_{6} \mathrm{H}_{4}$ & $4 b$ & $2 \mathrm{~h}$ & & 90 \\
\hline 16 & $\mathrm{C}_{10} \mathrm{H}_{7}$ & $4 b$ & $2 \mathrm{~h}$ & & 90 \\
\hline 17 & $\mathrm{Bn}$ & $4 b$ & $5 \mathrm{~min}$ & & 85 \\
\hline
\end{tabular}

${ }^{\mathrm{a}}$ Reaction conditions: See typical procedure; ${ }^{\mathrm{b}}$ Isolated yield.

\section{Experimental}

\subsection{General}

All reagents were obtained from commercial suppliers and used without further purification. Solvents were dried and distilled prior to use according to standard methods. The reaction was monitored by TLC on silica-gel plates (GF 254). ${ }^{1} \mathrm{H}-(300 \mathrm{MHz})$ and ${ }^{13} \mathrm{C}-\mathrm{NMR}(75 \mathrm{MHz})$ spectra were recorded on a Bruker APX-300 spectrometer at room temperature in $\mathrm{CDCl}_{3}$ using tetramethylsilane (TMS) as the internal standard. The coupling constants $J$ are given in Hz. All yields mentioned referred to isolated yields.

\subsection{Preparation of $\left(\mathrm{PPh}_{3}\right) \mathrm{AuCl}$}

The reaction was carried out in the absence of light. $\mathrm{SMe}_{2}(10 \mathrm{mmol})$ was added to a solution of $\mathrm{HAuCl}_{4} \cdot 2 \mathrm{H}_{2} \mathrm{O}(5 \mathrm{mmol})$ in $\mathrm{MeOH}(5 \mathrm{~mL})$, and the mixture was stirred for 10 minutes. The white precipitate $\left[\mathrm{AuCl}\left(\mathrm{SMe}_{2}\right)\right]$ from solution was then filtered, subsequently washed with $\mathrm{MeOH}, \mathrm{Et}_{2} \mathrm{O}$ and hexane, then dried under vacuum and used in the next step without further purification. Triphenylphosphine $(2 \mathrm{mmol})$ was added to a stirred solution of $\left[\mathrm{AuCl}\left(\mathrm{SMe}_{2}\right)\right](2 \mathrm{mmol})$ in $\mathrm{CH}_{2} \mathrm{Cl}_{2}$ $(15 \mathrm{~mL})$ under a nitrogen atmosphere. After stirring at room temperature for 30 minutes, the volume of the solution was reduced to $5 \mathrm{~mL}$ under reduced pressure, and then hexane $(20 \mathrm{~mL})$ was added, resulting in the precipitation of the complex. The solid was then filtered, washed with hexane and dried, resulting in the quantitative isolation of complex $\left[\left(\mathrm{PPh}_{3}\right) \mathrm{AuCl}\right]$ as a pale yellow solid $(97 \%)$. The complex was characterized only by ${ }^{31} \mathrm{P}-\mathrm{NMR}$ spectroscopy [42-43].

\subsection{Typical Procedure for the Synthesis of $\beta$-Enaminones and $\beta$-Enaminoesters}

The reaction was carried out without inert atmosphere and light protection. A mixture of $\left(\mathrm{PPh}_{3}\right) \mathrm{AuCl}(0.03 \mathrm{mmol}), \mathrm{AgOTf}(0.03 \mathrm{mmol})$ and 1,3 -dicarbonyl compound $(3 \mathrm{mmol})$ was stirred at room temperature for 5 minutes, then amine $(3 \mathrm{mmol})$ was added into the stirring solution. The reaction was monitored by TLC on silica-gel plates (GF 254). After the reaction was complete, the 
residue was diluted with water $(10 \mathrm{~mL})$ and extracted with ethyl acetate $(3 \times 10 \mathrm{~mL})$. The combined organic extracts were dried over anhydrous $\mathrm{Na}_{2} \mathrm{SO}_{4}$ and concentrated under reduced pressure after filtration. Further purification by flash chromatography gave the corresponding product.

(Z)-4-(4-Methoxyphenylamino)pent-3-en-2-one (3a). ${ }^{1} \mathrm{H}-\mathrm{NMR} \delta=12.31(\mathrm{~s}, 1 \mathrm{H}), 7.03-7.05(\mathrm{~m}, 2 \mathrm{H})$, 6.86-6.88 (m, 2H), $5.16(\mathrm{~s}, 1 \mathrm{H}), 3.79(\mathrm{~s}, 3 \mathrm{H}), 2.08(\mathrm{~s}, 3 \mathrm{H}), 1.90(\mathrm{~s}, 3 \mathrm{H}) ;{ }^{13} \mathrm{C}-\mathrm{NMR} \delta=195.78,161.15$, $157.60,131.35,126.54,114.13,96.79,55.33,28.99,19.54$.

(Z)-4-(Phenylamino)pent-3-en-2-one (3b). ${ }^{1} \mathrm{H}-\mathrm{NMR} \delta=12.44(\mathrm{~s}, 1 \mathrm{H}), 6.96-7.23$ (m, 5H), 5.09 $(\mathrm{s}, 1 \mathrm{H}), 1.99(\mathrm{~s}, 3 \mathrm{H}), 1.87(2,3 \mathrm{H}) ;{ }^{13} \mathrm{C}-\mathrm{NMR} \delta=195.45,159.62,138.18,128.59,124.98,124.05$, $97.18,28.66,19.30$

(Z)-4-(p-Tolylamino)pent-3-en-2-one (3c). ${ }^{1} \mathrm{H}-\mathrm{NMR} \delta=12.39(\mathrm{~s}, 1 \mathrm{H}), 7.07(\mathrm{~d}, 2 \mathrm{H}, J=8.4 \mathrm{~Hz}), 6.92$ $(\mathrm{d}, 2 \mathrm{H}, J=8.4 \mathrm{~Hz}), 5.12(\mathrm{~s}, 1 \mathrm{H}), 2.27(\mathrm{~s}, 3 \mathrm{H}), 2.04(\mathrm{~s}, 3 \mathrm{H}), 1.89(\mathrm{~s}, 3 \mathrm{H}) ;{ }^{13} \mathrm{C}-\mathrm{NMR} \delta=195.67,160.51$, $135.91,135.27,129.54,124.62,97.13,28.98,20.77,19.61$

(Z)-4-(4-Bromophenylamino)pent-3-en-2-one (3d). ${ }^{1} \mathrm{H}-\mathrm{NMR} \delta=12.38(\mathrm{~s}, 1 \mathrm{H}), 7.34(\mathrm{~d}, 2 \mathrm{H}, J=12 \mathrm{~Hz})$, $6.88(\mathrm{~d}, 2 \mathrm{H}, J=12 \mathrm{~Hz}), 5.13(\mathrm{~s}, 1 \mathrm{H}), 2.01(\mathrm{~s}, 3 \mathrm{H}), 1.90(\mathrm{~s}, 3 \mathrm{H}) ;{ }^{13} \mathrm{C}-\mathrm{NMR} \delta=196.31,159.32,137.74$, $132.02,125.83,118.43,98.16,29.13,19.70$.

(Z)-4-(4-Chlorophenylamino)pent-3-en-2-one (3e). ${ }^{1} \mathrm{H}-\mathrm{NMR} \delta=12.38(\mathrm{~s}, 1 \mathrm{H}), 7.18-7.22(\mathrm{~m}, 2 \mathrm{H})$, 6.92-6.96 (m, 2H), $5.12(\mathrm{~s}, 1 \mathrm{H}), 2.01(\mathrm{~s}, 3 \mathrm{H}), 1.89(\mathrm{~s}, 3 \mathrm{H}) ;{ }^{13} \mathrm{C}-\mathrm{NMR} \delta=196.3,159.5,137.2,129.1$, $125.6,98.0,29.1,19.7$.

(Z)-4-(Naphthalen-1-ylamino)pent-3-en-2-one (3f). ${ }^{1} \mathrm{H}-\mathrm{NMR} \delta=12.80(\mathrm{~s}, 1 \mathrm{H}), 8.02-8.05(\mathrm{~m}, 1 \mathrm{H})$, 7.83-7.86 (m, 1H), 7.72-7.75 (m, 1H), 7.49-7.55 (m, 2H), 7.39-7.44 (m, 1H), 7.23-7.26 (m, 1H), 5.31 (s, 1H), $2.12(\mathrm{~s}, 3 \mathrm{H}), 1.85(\mathrm{~s}, 3 \mathrm{H}) ;{ }^{13} \mathrm{C}-\mathrm{NMR} \delta=196.4,161.8,134.7,134.1,129.8,128.2,126.8,126.7$, $126.5,125.2,123.3,122.6,97.5,29.2,19.6$.

(Z)-4-Benzylamino)pent-3-en-2-one (3g). ${ }^{1} \mathrm{H}-\mathrm{NMR} \delta=11.17(\mathrm{~s}, 1 \mathrm{H}), 7.22-7.34(\mathrm{~m}, 5 \mathrm{H}), 5.03(\mathrm{~s}, 1 \mathrm{H})$, $4.44(\mathrm{~d}, 2 \mathrm{H}, J=6.3 \mathrm{~Hz}), 2.02(\mathrm{~s}, 3 \mathrm{H}), 1.88(2,3 \mathrm{H}) ;{ }^{13} \mathrm{C}-\mathrm{NMR} \delta=195.1,162.9,137.8,128.6,127.2$, $126.5,95.7,77.4,77.0,76.6,46.4,28.7,18.7$.

(Z)-4-(Butylamino)pent-3-en-2-one (3h). ${ }^{1} \mathrm{H}-\mathrm{NMR} \delta=10.80(\mathrm{~s}, 1 \mathrm{H}), 4.87$ (s, 1H), 3.15 (q, 2H, $\left.J=6.6 \mathrm{~Hz}\right)$, $1.91(\mathrm{~s}, 3 \mathrm{H}), 1.84(\mathrm{~s}, 3 \mathrm{H}), 1.44-1.54(\mathrm{~m}, 2 \mathrm{H}), 1.27-1.39(\mathrm{~m}, 2 \mathrm{H}), 0.85(\mathrm{q}, 3 \mathrm{H}, J=7.2 \mathrm{~Hz}) ;{ }^{13} \mathrm{C}-\mathrm{NMR}$ $\delta=194.3,162.9,94.7,42.4,31.9,28.5,19.8,19.7,18.6,13.5,13.5$.

(Z)-4-(Allylamino)pent-3-en-2-one (3i). ${ }^{1} \mathrm{H}-\mathrm{NMR} \delta=10.70(\mathrm{~s}, 1 \mathrm{H}), 5.64-5.73(\mathrm{~m}, 2 \mathrm{H}), 4.97-5.07(\mathrm{~m}, 2 \mathrm{H})$, $4.83(\mathrm{~s}, 3 \mathrm{H}), 3.67-3.71(\mathrm{~m}, 2 \mathrm{H}), 1.82(\mathrm{~s}, 1 \mathrm{H}), 1.74(\mathrm{~s}, 1 \mathrm{H}) ;{ }^{13} \mathrm{C}-\mathrm{NMR} \delta=194.7,162.9,133.9,115.7$, 95.4, 44.8, 28.6, 18.3 .

(Z)-4-(2-Hydroxyethylamino)pent-3-en-2-one (3j). ${ }^{1} \mathrm{H}-\mathrm{NMR} \delta=10.71(\mathrm{~s}, 1 \mathrm{H}), 5.02(\mathrm{~s}, 1 \mathrm{H}), 4.83$ $(\mathrm{s}, 1 \mathrm{H}), 3.60(\mathrm{t}, 2 \mathrm{H}, J=5.4 \mathrm{~Hz}), 3.27(\mathrm{t}, 2 \mathrm{H}, J=5.4 \mathrm{~Hz}), 1.83(\mathrm{~s}, 6 \mathrm{H}) ;{ }^{13} \mathrm{C}-\mathrm{NMR} \delta=194.3,164.1$, 95.3, 61.0, 45.3, 28.3, 18.9 . 
(Z)-Ethyl 3-(4-Methoxyphenylamino)but-2-enoate (5a). ${ }^{1} \mathrm{H}-\mathrm{NMR} \delta=10.15(\mathrm{~s}, 1 \mathrm{H}), 6.97-7.00(\mathrm{~m}, 2 \mathrm{H})$, 6.80-6.83 (m, 2H), 4.62 (s, 1H), 4.07-4.14 (q, 2H, J=7.0 Hz), 3.75 (s, 3H), 1.85 (s, 3H), 1.24 (t, 3H, $J=7.0 \mathrm{~Hz}) ;{ }^{13} \mathrm{C}-\mathrm{NMR} \delta=170.3,159.7,157.2,131.9,126.5,113.9,84.5,58.4,55.1,19.9,14.4$.

(Z)-Ethyl 3-(Phenylamino)but-2-enoate (5b). ${ }^{1} \mathrm{H}-\mathrm{NMR} \quad \delta=10.43(\mathrm{~s}, 1 \mathrm{H}), 7.27-7.32(\mathrm{~m}, 2 \mathrm{H})$, $7.05-7.15$ (m, 3H), 4.70 (s, 1H), 4.11-4.18 (q, 2H, J=7.2 Hz), 1.97 (s, 3H), 1.27 (t, 3H, $J=7.2 \mathrm{~Hz}$ ); ${ }^{13} \mathrm{C}-\mathrm{NMR} \delta=170.3,158.8,139.2,120.0,124.8,124.2,86.0,58.6,20.2,14.5$.

(Z)-Ethyl 3-(p-Tolylamino)but-2-enoate (5c). ${ }^{1} \mathrm{H}-\mathrm{NMR} \delta=10.32(\mathrm{~s}, 1 \mathrm{H}), 7.10-7.13$ (m, 2H), 6.95-6.98 (m, 2H), $4.67(\mathrm{~s}, 1 \mathrm{H}), 4.14$ (q, 2H, J=7.2 Hz), $2.32(\mathrm{~s}, 3 \mathrm{H}), 1.94(\mathrm{~s}, 3 \mathrm{H}), 1.28$ (t, 3H, $J=7.2 \mathrm{~Hz}) .{ }^{13} \mathrm{C}-\mathrm{NMR} \delta=170.3,159.3,136.6,134.7,129.5,124.6,85.3,58.6,20.8,20.1,14.5$.

(Z)-Ethyl 3-(4-Bromophenylamino)but-2-enoate (5d). ${ }^{1} \mathrm{H}-\mathrm{NMR} \delta=10.34(\mathrm{~s}, 1 \mathrm{H}), 7.34-7.37(\mathrm{~m}, 2 \mathrm{H})$, 6.88-6.89 (m, 2H), 4.67 (s, 1H), 4.06-4.13 (q, 2H, J=7.2 Hz), 1.93 (s, 3H), 1.23 (t, 3H, J=7.2 Hz); ${ }^{13} \mathrm{C}-\mathrm{NMR} \delta=170.2,158.0,138.4,132.0,125.5,117.7,87.0,58.8,20.1,14.5$.

(Z)-Ethyl 3-(4-Chlorophenylamino)but-2-enoate (5e). ${ }^{1} \mathrm{H}-\mathrm{NMR} \delta=10.36(\mathrm{~s}, 1 \mathrm{H}), 7.24-7.27(\mathrm{~m}, 2 \mathrm{H})$, 6.97-7.00 (m, 2H), $4.70(\mathrm{~s}, 1 \mathrm{H}), 4.12(\mathrm{q}, 2 \mathrm{H}, J=7.2 \mathrm{~Hz}), 1.95(\mathrm{~s}, 3 \mathrm{H}), 1.26(\mathrm{t}, 3 \mathrm{H}, J=7.2 \mathrm{~Hz})$. ${ }^{13} \mathrm{C}-\mathrm{NMR} \delta=170.3,158.2,137.9,130.1,129.1,125.3,116.1,86.8,58.8,20.1,14.5$.

(Z)-Ethyl 3-(Naphthalen-1-ylamino)but-2-enoate (5f). ${ }^{1} \mathrm{H}-\mathrm{NMR} \delta=10.68(\mathrm{~s}, 1 \mathrm{H}), 8.10-8.13(\mathrm{~m}, 1 \mathrm{H})$, 7.87-7.90 (m, 1H), 7.74-7.77 (m, 1H), 7.52-7.57 (m, 2H), 7.42-7.47 (m, 1H), 7.27-7.29 (m, 1H), 4.88 $(\mathrm{s}, 1 \mathrm{H}), 4.27(\mathrm{q}, 2 \mathrm{H}, J=7.2 \mathrm{~Hz}), 1.89(\mathrm{~s}, 3 \mathrm{H}), 1.37(\mathrm{t}, 3 \mathrm{H}, J=7.2 \mathrm{~Hz}) .{ }^{13} \mathrm{C}-\mathrm{NMR} \delta=170.7,160.4$, $135.3,134.2,130.4,128.2,126.7,126.5,126.4,125.3,123.5,122.7,85.8,58.8,20.0,14.7$.

(Z)Ethyl 3-(Benzylamino)but-2-enoate (5g). ${ }^{1} \mathrm{H}-\mathrm{NMR} \delta=8.96(\mathrm{~s}, 1 \mathrm{H}), 7.21-7.29(\mathrm{~m}, 5 \mathrm{H}), 4.53$ $(\mathrm{s}, 1 \mathrm{H}), 4.34(\mathrm{~s}, 2 \mathrm{H}), 4.04-4.11(\mathrm{~m}, 3 \mathrm{H}), 1.84(\mathrm{t}, 3 \mathrm{H}, J=3.6 \mathrm{~Hz}), 1.18-1.24(\mathrm{~m}, 3 \mathrm{H}) ;{ }^{13} \mathrm{C}-\mathrm{NMR}$ $\delta=170.4,161.7,138.7,128.7,127.2,126.6,83.1,58.2,46.6,19.2,14.5$.

(Z)-Ethyl 3-(Butylamino)but-2-enoate (5h). ${ }^{1} \mathrm{H}-\mathrm{NMR} \delta=8.46(\mathrm{~s}, 1 \mathrm{H}), 4.31(\mathrm{~s}, 1 \mathrm{H}), 3.96(\mathrm{q}, 2 \mathrm{H}$, $J=7.2 \mathrm{~Hz}), 3.06-3.13(\mathrm{~m}, 2 \mathrm{H}), 1.81(\mathrm{~s}, 3 \mathrm{H}), 1.40-1.49(\mathrm{~m}, 2 \mathrm{H}), 1.24-1.36(\mathrm{~m}, 2 \mathrm{H}), 1.13(\mathrm{t}, 3 \mathrm{H}$, $J=7.2 \mathrm{~Hz}), 0.83(\mathrm{t}, 3 \mathrm{H}, J=7.2 \mathrm{~Hz}) ;{ }^{13} \mathrm{C}-\mathrm{NMR} \delta=170.4,161.7,81.5,57.9,42.5,32.3,19.8,19.1$, 14.4, 13.6 .

(Z)-Ethyl 3-(Allylamino)but-2-enoate (5i). ${ }^{1} \mathrm{H}$ NMR (CDCl3, $\left.300 \mathrm{MHz}\right) \delta=8.61(\mathrm{~s}, 1 \mathrm{H}), 5.74-5.85$ $(\mathrm{m}, 1 \mathrm{H}), 5.06-5.19(\mathrm{~m}, 2 \mathrm{H}), 4.42(\mathrm{~s}, 1 \mathrm{H}), 4.02(\mathrm{q}, 2 \mathrm{H}, J=7.2 \mathrm{~Hz}), 3.75-3.80(\mathrm{~m}, 2 \mathrm{H}), 1.85(\mathrm{~s}, 3 \mathrm{H})$, $1.18(\mathrm{t}, 3 \mathrm{H}, J=7.2 \mathrm{~Hz}) ;{ }^{13} \mathrm{C} \mathrm{NMR}\left(\mathrm{CDCl}_{3}, 75 \mathrm{MHz}\right) \delta=170.4,161.7,134.7,115.6,82.6,58.1,45.0$, $18.9,14.5$.

(Z)-Ethyl 3-(2-Hydroxyethylamino)but-2-enoate (5j). ${ }^{1} \mathrm{H}$ NMR $\left(\mathrm{CDCl}_{3}, 300 \mathrm{MHz}\right) \delta=8.61(\mathrm{~s}, 1 \mathrm{H})$, 4.44 (s, 1H), 4.04 (q, 2H, $J=7.2 \mathrm{~Hz}), 3.70$ (t, 2H, $J=5.4 \mathrm{~Hz}), 3.13$ (br, 1H), 3.33 (q, 2H, $J=5.4 \mathrm{~Hz}$ ), $1.91(\mathrm{~s}, 1 \mathrm{H}), 1.21(\mathrm{t}, 3 \mathrm{H}, J=7.2 \mathrm{~Hz}) ;{ }^{13} \mathrm{C} \mathrm{NMR}\left(\mathrm{CDCl}_{3}, 75 \mathrm{MHz}\right) \delta=170.7,162.1,82.6,61.8,58.4$, $45.0,19.5,14.5$. 
Ethyl 2-(4-Methoxyphenylamino)cyclopent-1-enecarboxylate (5k). ${ }^{1} \mathrm{H}-\mathrm{NMR} \delta=9.32(\mathrm{~s}, 1 \mathrm{H}), 6.90-6.93$ (m, 2H), 6.74-6.77 (m, 2H) , 4.14 (q, 2H, $J=7.2 \mathrm{~Hz}), 3.69$ (s, 1H), 2.49-2.60 (m, 4H), 1.71-1.80 (m, 2H), 1.25 (t, 3H, $J=7.2 \mathrm{~Hz}) ;{ }^{13} \mathrm{C}-\mathrm{NMR} \delta=168.3,161.4,156.1,133.6,123.3,114.1,95.9,58.6,55.2$, $33.1,28.8,21.5,14.6$.

Ethyl 2-(Phenylamino) cyclopent-1-enecarboxylate (5l). ${ }^{1} \mathrm{H}-\mathrm{NMR} \delta=9.65(\mathrm{~s}, 1 \mathrm{H}), 7.24-7.30(\mathrm{~m}, 2 \mathrm{H})$, 6.99-7.05 (m, 3H) , 4.21 (q, 2H, $J=7.2 \mathrm{~Hz}), 2.79$ (t, 2H, $J=7.2 \mathrm{~Hz}), 2.58(\mathrm{t}, 2 \mathrm{H}, J=7.2 \mathrm{~Hz}), 1.86(\mathrm{~m}$, 2H), $1.32(\mathrm{t}, 3 \mathrm{H}, J=7.2 \mathrm{~Hz}) ;{ }^{13} \mathrm{C}-\mathrm{NMR} \delta=168.4,160.3,140.6,129.1,123.0,120.6,97.6,58.9,33.6$, $28.7,21.7,14.6$.

Ethyl 2-(p-Tolylamino)cyclopent-1-enecarboxylate $(\mathbf{5 m}) .{ }^{1} \mathrm{H}-\mathrm{NMR} \delta=9.55(\mathrm{~s}, 1 \mathrm{H}), 7.05-7.08(\mathrm{~m}$, 2H), 6.92-6.94 (m, 2H), 4.21 (q, 2H, $J=7.2 \mathrm{~Hz}), 2.73$ (t, 2H, $J=7.2 \mathrm{~Hz}), 2.57(\mathrm{t}, 2 \mathrm{H}, J=7.2 \mathrm{~Hz}), 2.29$ $(\mathrm{s}, 3 \mathrm{H}), 1.84(\mathrm{~m}, 2 \mathrm{H}), 1.31(\mathrm{t}, 3 \mathrm{H}, J=7.2 \mathrm{~Hz}) ;{ }^{13} \mathrm{C}-\mathrm{NMR} \delta=168.4,160.8,138.1,132.7,129.6,121.0$, $96.8,58.8,33.4,28.7,21.7,20.6,14.6$.

Ethyl 2-(4-Bromophenylamino) cyclopent-1-enecarboxylate (5n). ${ }^{1} \mathrm{H}-\mathrm{NMR} \delta=9.61(\mathrm{~s}, 1 \mathrm{H}), 7.30-7.35$ $(\mathrm{m}, 2 \mathrm{H}), 6.83-6.88(\mathrm{~m}, 2 \mathrm{H}), 4.11(\mathrm{q}, 2 \mathrm{H}, J=7.2 \mathrm{~Hz}), 2.72(\mathrm{t}, 2 \mathrm{H}, J=7.2 \mathrm{~Hz}), 2.53(\mathrm{t}, 2 \mathrm{H}, J=7.2 \mathrm{~Hz})$, $1.83(\mathrm{~m}, 2 \mathrm{H}), 1.28(\mathrm{t}, 3 \mathrm{H}, J=7.2 \mathrm{~Hz}) ;{ }^{13} \mathrm{C}-\mathrm{NMR} \delta=168.3,159.4,139.7,132.0,121.7,115.4,98.6$, 59.0, 33.5, 28.6, 21.7, 14.6.

Ethyl 2-(4-Chlorophenylamino)cyclopent-1-enecarboxylate (5o). ${ }^{1} \mathrm{H}-\mathrm{NMR} \delta=9.60(\mathrm{~s}, 1 \mathrm{H}), 7.16-7.19$ $(\mathrm{m}, 2 \mathrm{H}), 6.89-6.92(\mathrm{~m}, 2 \mathrm{H}), 4.16(\mathrm{q}, 2 \mathrm{H}, J=7.2 \mathrm{~Hz}), 2.72(\mathrm{t}, 2 \mathrm{H}, J=7.2 \mathrm{~Hz}), 2.53(\mathrm{t}, 2 \mathrm{H}, J=7.2 \mathrm{~Hz})$, $1.83(\mathrm{~m}, 2 \mathrm{H}), 1.27(\mathrm{t}, 3 \mathrm{H}, J=7.2 \mathrm{~Hz}) ;{ }^{13} \mathrm{C}-\mathrm{NMR} \delta=168.3,159.5,139.2,129.0,127.9,121.4,98.4$, $59.0,33.5,28.6,21.6,14.5$.

Ethyl 2-(Naphthalen-1-ylamino)cyclopent-1-enecarboxylate $(\mathbf{5 p}) .{ }^{1} \mathrm{H}-\mathrm{NMR} \delta=10.02(\mathrm{~s}, 1 \mathrm{H}), 8.18-8.21$ (m, 1H), 7.85-7.88 (m, 1H), 7.52-7.66 (m, 3H), 7.38-7.44 (m, 1H), 7.22-7.25 (m, 1H), 7.27-7.29 $(\mathrm{m}, 1 \mathrm{H}), 4.33(\mathrm{q}, 2 \mathrm{H}, J=7.2 \mathrm{~Hz}), 2.69(\mathrm{~m}, 4 \mathrm{H}), 1.86(\mathrm{~m}, 2 \mathrm{H}), 1.40(\mathrm{t}, 3 \mathrm{H}, J=7.2 \mathrm{~Hz}) ;{ }^{13} \mathrm{C}-\mathrm{NMR}$ $\delta=168.8,161.6,136.3,134.3,128.3,126.4,126.3,125.4,124.6,122.0,119.0,97.7,59.0,33.4,29.0$, 21.6, 14.8 .

Ethyl 2-(Benzylamino)cyclopent-1-enecarboxylate (5q). ${ }^{1} \mathrm{H}-\mathrm{NMR} \delta=7.80(\mathrm{~s}, 1 \mathrm{H}), 7.25-7.35(\mathrm{~m}, 5 \mathrm{H})$, $4.38(\mathrm{~d}, 2 \mathrm{H}, J=6.6 \mathrm{~Hz}), 4.16(\mathrm{q}, 2 \mathrm{H}, J=7.2 \mathrm{~Hz}), 2.54(\mathrm{q}, 4 \mathrm{H}, J=7.2 \mathrm{~Hz}), 1.81(\mathrm{~m}, 2 \mathrm{H}), 1.28(\mathrm{t}, 3 \mathrm{H}$, $J=7.2 \mathrm{~Hz}) ;{ }^{13} \mathrm{C}-\mathrm{NMR} \delta=168.4,164.5,139.2,128.6,127.2,126.7,93.4,58.4,48.3,32.0,29.1,20.8,14.7$.

\section{Conclusions}

In summary, we have developed an efficient method for the synthesis of $\beta$-enaminones and $\beta$-enaminoesters via reaction of 1,3-dicarbonyl compounds with various primary amines under solvent-free conditions catalyzed by $\left[\left(\mathrm{PPh}_{3}\right) \mathrm{AuCl}\right] / \mathrm{AgOTf}$. This methodology affords various $\beta$-enaminones and $\beta$-enaminoesters derivatives in good to excellent yields. Moreover, the method has the advantage of easy manipulation and mild reaction conditions. 


\section{Acknowledgements}

We gratefully acknowledge the National Natural Science Foundation of China (20832001, 20972065, 21074054, 21172106) and the National Basic Research Program of China (2010CB923303) for their financial support.

\section{References and Notes}

1. Reddy, G.J.; Latha, D.; Thirupathaiah, C.; Rao, K.S. A facile synthesis of 2,3-disubstituted-6arylpyridines from enaminones using montmorillonite K10 as solid acid support. Tetrahedron Lett. 2005, 46, 301-302.

2. Negri, G.; Kascheres, C.; Kascheres, A.J. Recent developments in the chemistry of enaminones. J. Heterocycl. Chem. 2004, 41, 461-491.

3. Abdelkhalik, M.M.; Eltoukhy, A.M.; Agamy, M.; Elnagdi, M.H. A General and Efficient Method for the Preparation of $\beta$-Enamino Ketones and Esters Catalyzed by Indium Tribromide. J. Heterocycl. Chem. 2004, 41, 431-434.

4. Michael, J.P.; Koning, C.B.; Hosken, G.D.; Stanbury, T.V. Reformatsky reactions with $\mathrm{N}$-arylpyrrolidine-2-thiones: Synthesis of tricyclic analogues of quinolone antibacterial agents. Tetrahedron 2001, 57, 9635-9648.

5. Azzaro, M.; Geribaldi, S.; Videau, B. Use of Boron Trifluoride Etherate in the Preparation of 2-Amino-1-alkenyl Ketones from $\beta$-Diketones and Low-Boiling Amines. Synthesis 1981, 880-881.

6. Dannhardt, G.; Bauer, A.; Nowe, U. Synthesis and Pharmacological Activity of Enaminones which Inhibit both Bovine Cyclooxygenase and 5-Lipoxygenase. J. Prakt. Chem. 1998, 340, 256-263.

7. Boger, D.L.; Ishizaki, T.; Wysocki, J.R.J.; Munk, S.A.; Kitos, P.A.; Suntornwat, O. Total synthesis and evaluation of $( \pm)$-N-(tert-butoxycarbonyl)-CBI, $( \pm)-\mathrm{CBI}-\mathrm{CDPI} 1$, and $( \pm)-\mathrm{CBI}-$ CDPI2: CC-1065 functional agents incorporating the equivalent 1,2,9,9a-tetrahydrocyclopropa[1,2c] benz[1,2-e]indol-4-one (CBI) left-hand subunit. J. Am. Chem. Soc. 1989, 111, 6461-6463.

8. Haycock-Lewandowski, S.J.; Wilder, A.; Ahman, J. Development of a Bulk Enabling Route to Maraviroc (UK-427,857), a CCR-5 Receptor Antagonist. J. Org. Process Res. Dev. 2008, 12, 1094-1103.

9. Cimarelli, C.; Palmieri, G. Stereoselective Reduction of Enantiopure $\beta$-Enamino Esters by Hydride: A Convenient Synthesis of Both Enantiopure $\beta$-Amino Esters. J. Org. Chem. 1996, 61, 5557-5563.

10. Potin, D.; Dumas, F.; Angelo, J.D. New chiral auxiliaries: Their use in the asymmetric hydrogenation of $\beta$-acetamidocrotonates. J. Am. Chem. Soc. 1990, 112, 3483-3486.

11. Cimarelli, C.; Palmieri, G.; Volpini, E. An Improved Synthesis of Enantiopure $\beta$-Amino Acids. Synth. Commun. 2001, 31, 2943-2953.

12. Beholz, L.G.; Benovsky, R.; Ward, D.L.; Bata, N.S.; Stille, J.R. Formation of Dihydropyridoneand Pyridone-Based Peptide Analogs through Aza-Annulation of $\beta$-Enamino Ester and Amide Substrates with $\alpha$-Amido Acrylate Derivatives. J. Org. Chem. 1997, 62, 1033-1042. 
13. Nakamura, I.; Yamamoto, Y. Transition-Metal-Catalyzed Reactions in Heterocyclic Synthesis. Chem. Rev. 2004, 104, 2127-2198.

14. Chaaban, I.; Greenhill, J.V.; Akhtar, P. Enaminones in the mannich reaction. Part 2. Further investigations of internal mannich reactions. J. Chem. Soc. Perkin Trans. I 1979, 1593-1596.

15. Figueiredo, L.J.O.; Kascheres, C. Quinone Diazides and Enaminones as a Source of New Azo Compounds with Potential Nonlinear Optical Properties. J. Org. Chem. 1997, 62, 1164-1167.

16. Aceña, J.L.; Arjona, O.; Mañas, R.; Plumet, J. Unexpected One-Pot Epoxy Sulfone-Enaminone Transformation. Synthesis of $5 \alpha$-Carba- $\beta$-mannopyranosylamine. J. Org. Chem. 2000, 65, 2580-2582.

17. Li, G.; Watson, K.; Buckheit, R.W.; Zhang, Y. Total Synthesis of Anibamine, a Novel Natural Product as a Chemokine Receptor CCR5 Antagonist. Org. Lett. 2007, 9, 2043-2046.

18. Calle, M.; Calvo, L.A.; Ortega, A.G.; Gonzalez-Nogal, A.M. Silylated $\beta$-enaminones as precursors in the regioselective synthesis of silyl pyrazoles. Tetrahedron 2006, 62, 611-618.

19. Ferraz, H.M.C.; Pereira, F.L.C.; Leite, F.S.; Nuns, M.R.S.; Payret-Arrua, M.E. Synthesis of $\mathrm{N}$-substituted pyrrole and tetrahydroindole derivatives from alkenyl $\beta$-dicarbonyl compounds. Tetrahedron 1999, 55, 10915-10924.

20. Khosropour, A.R.; Khodaei, M. A mild, efficient and environmentally friendly method for the regio- and chemoselective synthesis of enaminones using $\mathrm{Bi}(\mathrm{TFA})_{3}$ as a reusable catalyst in aqueous media. Tetrahedron Lett. 2004, 45, 1725-1728.

21. Zhang, Z.H.; Li, T.S.; Li, J.J. Synthesis of enaminones and enamino esters catalysed by $\mathrm{ZrOCl}_{2} \cdot 8 \mathrm{H}_{2} \mathrm{O}$. Catal. Commun. 2007, 8, 1615-1620.

22. Li, G.C. Simple and Efficient Synthesis of 3-Aminopropenones and 3-Aminopropenoates Catalyzed by Copper (II) Nitrate Trihydrate under Solvent-Free Conditions. Monatsh. Chem. 2008, 139, 789-792.

23. Xu, S.L.; Li, C.P.; Li, J.H. Solid-State Synthesis of $\beta$-Enamino Ketones from Solid 1,3-Dicarbonyl Compounds and Ammonium Salts or Amines. Synlett 2009, 5, 818-822.

24. Chen, X.; She, J.; Shang, Z.C.; Wu, J.; Wu, H.F.; Zhang, P.Z. Synthesis of Pyrazoles, Diazepines, Enaminones, and Enamino Esters Using 12-Tungstophosphoric Acid as a Reusable Catalyst in Water. Synthesis 2008, 21, 3478-3486.

25. Rafiee, E.; Joshaghani, M.; Eavania, S.; Rashidzadeh, S. A revision for the synthesis of $\beta$-enaminones in solvent free conditions: efficacy of different supported heteropoly acids as active and reusable catalysts. Green Chem. 2008, 10, 982-989.

26. Sridharan, V.; Avendaño, C.; Menéndez, J.C. General, Mild and Efficient Synthesis of $\beta$-Enaminones Catalyzed by Ceric Ammonium Nitrate. Synlett 2007, 6, 881-884.

27. Arcadi, A.; Bianchi, G.; Giuseppe, S.D.; Marinelli, F. Gold catalysis in the reactions of 1,3-dicarbonyls with nucleophiles. Green Chem. 2003, 5, 64-67.

28. Zhang, Z.H.; Yin, L.; Wang, Y.M. Tandem $\beta$-Enamino Ester Formation and Cyclization with o-Alkynyl Anilines Catalyzed by $\mathrm{InBr}_{3}$ : Efficient Synthesis of $\beta$-(N-Indolyl)- $\alpha, \beta$-unsaturated Esters. Adv. Synth. Catal. 2006, 348, 184-190.

29. Krishna, P.R.; Sekhar, E.R. p-Toluenesulfonylmethyl Isocyanide (TosMIC) and Indium Manifold Strategy to Access $\beta$-Keto-(E)-enamino Esters from 1,3-Dicarbonyl Compounds. Adv. Synth. Catal. 2008, 350, 2871-2876. 
30. Kidwai, M.; Bhardwaj, S.; Mishra, N.K.; Bansal, V.; Kumar, A.; Mozumdar, S. A novel method for the synthesis of $\beta$-enaminones using $\mathrm{Cu}$-nanoparticles as catalyst. Catal. Commun. 2009, 10, $1514-1517$.

31. Harrad, M.A.; Outtouch, R.; Ali, M.A.; Firdoussi, L.E.; Karim, A.; Roucoux, A. $\left.\mathrm{Ca}_{(\mathrm{CF}} \mathrm{COO}\right)_{2}$ : An efficient Lewis acid catalyst for chemo- and regio-selective enamination of $\beta$-dicarbonyl compounds. Catal. Commun. 2010, 11, 442-446.

32. Zhang, Z.H.; Ma, Z.C.; Mo, L.P. Enamination of 1,3-dicarbonyl compounds catalyzed by tin tetrachloride. Indian J. Chem. 2007, 46B, 535-539.

33. Stefani, H.A.; Costa, I. M.; Silva, D.D. An Easy Synthesis of Enaminones in Water as Solvent. Synthesis 2000, 1526-1528.

34. Khodaei, M.M.; Khosropour, A.R.; Kookhazadeh, M. A novel enamination of $\beta$-dicarbonyl compounds catalyzed by $\mathrm{Bi}(\mathrm{TFA})_{3}$ immobilized on molten TBAB. Can. J. Chem. 2005, 83, 209-212.

35. Hashmi, A.S.K. Gold-Catalyzed Organic Reactions. Chem. Rev. 2007, 107, 3180-3211.

36. Nieto-Oberhuber, C.; Muñoz, M.P.; Buñuel, E.; Nevado, C.; Cárdenas, D.J.; Echavarren, A.M. Cationic Gold(I) Complexes: Highly Alkynophylic Catalysts for the Exo- and Endo-Cyclization of Enynes. Angew. Chem. Int. Ed. Engl. 2004, 43, 2402-2406.

37. Brandys, M.C.; Jennings, M.C.; Puddephatt, R.J. Luminescent gold(I) macrocycles with diphosphine and 4,4'-bipyridyl ligands. J. Chem. Soc. Dalton Trans. 2000, 4601-4606.

38. Ito, H.; Saito, T.; Miyahara, T.; Zhong, C.; Sawamura, M. Gold(I) Hydride Intermediate in Catalysis: Dehydrogenative Alcohol Silylation Catalyzed by Gold(I) Complex. Organometallics 2009, 28, 4829-4840.

39. Metzger, J.O. Solvent-Free Organic Syntheses. Angew. Chem. Int. Ed. Engl. 1998, 37, 2975-2978.

40. Tanaka, K.; Toda, F. Solvent-Free Organic Synthesis. Chem. Rev. 2000, 100, 1025-1074.

41. Cave, G.W.V.; Raston, C.L.; Scotta, J.L. Recent advances in solventless organic reactions: Towards benign synthesis with remarkable versatility. Chem. Commun. 2001, 37, 2159-2169.

42. Mézailles, N.; Ricard, L.; Gagosz, F. Phosphine Gold(I) Bis-(trifluoromethanesulfonyl)imidate Complexes as New Highly Efficient and Air-Stable Catalysts for the Cycloisomerization of Enynes. Org. Lett. 2005, 7, 4133-4136.

43. Sanguramath, R.A.; Hooper, T.N.; Butts, C.P.; Green, M.; McGrady, J.E.; Russell, C.A. The Interaction of Gold(I) Cations with 1,3-Dienes. Angew. Chem. Int. Ed. Engl. 2011, 50, 7592-7595.

Sample Availability: Samples of the compounds are available from the authors.

(C) 2012 by the authors; licensee MDPI, Basel, Switzerland. This article is an open access article distributed under the terms and conditions of the Creative Commons Attribution license (http://creativecommons.org/licenses/by/3.0/). 